\title{
Exome sequencing and pathogenicity- network analysis of five French families implicate mTOR signalling and autophagy in familial sarcoidosis
}

\author{
To the Editor:
}

Sarcoidosis is a complex disease characterised by the presence of epithelioid non-caseating granulomatous inflammation affecting multiple organs and whose aetiology has been related to microbial, environmental and genetic factors $[1,2]$. However, no single antigenic trigger has been identified, although associations with Propionibacterium acnes and mycobacteria pathogen-associated molecular pattern, deposition of serum amyloid A, and exposure to inorganic particles and insecticides have been suggested [3-5]. Occurrence of familial cases suggests that genetic variation contributes to disease pathogenesis with a heritability of about 39\% [6]. Despite intensive genome-wide association studies, no single nucleotide polymorphism is, as yet, able to explain the "missing heritability" in the disease, especially for non-resolving, non-Löfgren syndrome sarcoidosis [5-7].

To overcome the issue of missing heritability in multifactorial diseases, different strategies to unravel the genetic complexity have been proposed. These include: 1) a select focus on sequencing high susceptibility individuals with young age-at-onset, or with positive family history [8]; 2) analysing a cohort of stringent, well-phenotyped families with accessible sibships; 3) pooling of rare variants for analysis; and 4) elucidating the gene regulatory network of identified disease genes by functional pathway gene analyses $[7,9]$. Here, we incorporated the above-mentioned strategic guidelines and genotyped by whole exome sequencing a cohort of five clinically screened families with non-Löfgren syndrome sarcoidosis. Clinical diagnosis criteria were according to American Thoracic Society/European Respiratory Society/World Association of Sarcoidosis and other Granulomatous Disorders guidelines [10] and diagnosis of sarcoidosis in all cases was based on histological confirmation of either lymph nodes and/or bronchial biopsies. Families F1 to F5 were collected through the national SARCFAM observational project, whose primary objective was to establish a clinical and genetic database on sporadic and familial sarcoidosis [8]. The five families, presenting an autosomal dominant pattern of inheritance, included a total of 14 affected individuals together with eight family-matched non-affected first degree relatives (figure 1a), which allowed a genotype comparison between affected cases and healthy controls within each family. Such a genomic subtraction between cases and internal controls of the same family would allow genetic triggers associated with the inherited predisposition to sarcoidosis to be highlighted, and assuming or considering an autosomal mode of transmission, select for highly penetrant variants/genes.

Next, we sought to pool susceptibility variants prioritised from a series of functional prediction software programs to accentuate the cumulative deleterious effects of variants found only in probands but not in internal healthy controls. Thus, variants subtracted between affected family members and healthy family internal controls were selected when they met one of the following criteria: minor allele frequency lower than $0.05(5 \%)$, and a pathogenic score either by SIFT $(<0.05)$, POLYPHENv2 $(>0.400)$, MutationTaster or Alamut Visual Interactive Biosoftware. The variants per family were then pooled together from all five families, which resulted in a total of 227 disease susceptibility variants in 192 genes, including 223 (88.9\%) missense variants, two $(0.7 \%)$ splicing sites, nine (3.6\%) in-frame deletion/insertion, one (0.3\%) start-stop, and eight $(3.2 \%)$ nonsense variants.

@ERSpublications

Sarcoidosis is a multifactorial disease induced by environmental and genetic factors. Whole exome sequencing in $\mathbf{5}$ familial cases of the disease suggest that genetic defects in Racl and mTor functional hubs may be relevant to sarcoidosis pathogenesis. http://bit.ly/2xooHx3

Cite this article as: Calender A, Lim CX, Weichhart T, et al. Exome sequencing and pathogenicitynetwork analysis of five French families implicate mTOR signalling and autophagy in familial sarcoidosis. Eur Respir J 2019; 54: 1900430 [https://doi.org/10.1183/13993003.00430-2019]. 
a)

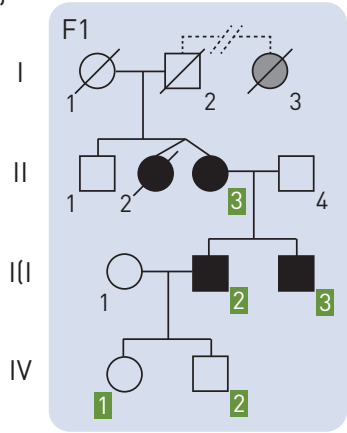

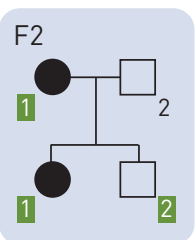

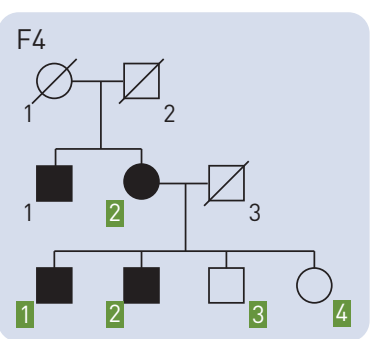

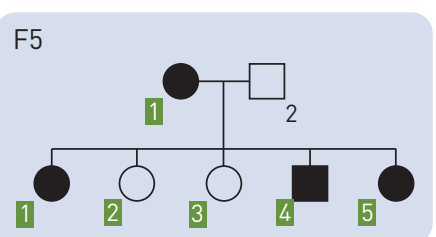

b)
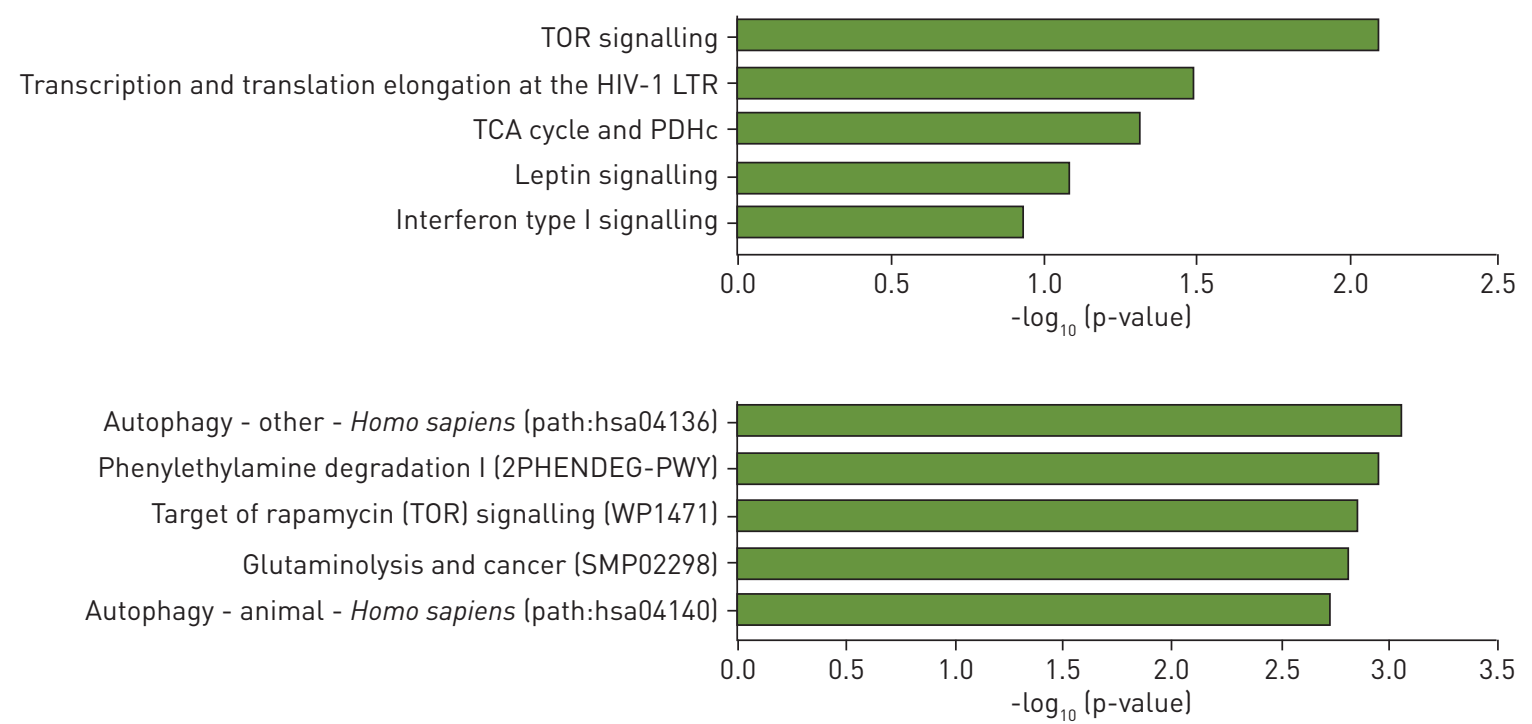

FIGURE 1 a) Families (F1to F5), with a total of 14 affected (filled symbols) and eight unaffected (clear symbols) individuals were analysed. The labelled green squares indicate the analysed individuals. All protocols involving study participants outlined in this manuscript have been approved by the French CCP LYON SUD EST-2 Ethical Committee (REF IRB 00009118). All participants provided written consent and gave permission to use their DNA for research purposes. b) Exome sequencing and variant calling were performed as described previously [12]. Two independent NGS sequencing rounds were performed on the whole series (F1 to F5) and only the variants identified recurrently were kept. Gene identifiers from the selected 227 variants derived by the Ensembl Variant Effect Predictor were then used for gene set over-representation pathway analyses. Gene identifiers were mapped to the top five ranked pathway gene sets found in WikiPathways 2016, and ConsensusPathDB.

To control for variant classification and annotation bias, we used Variant Effect Predictor to determine the functional effect of the 227 identified susceptibility variants on transcripts curated in Ensembl's database (www.ensembl.org/) mapped $20000 \mathrm{bp}$ upstream and downstream in the Ensembl human GRCh38 Assembly database. We then took all the HUGO gene identifiers encoding the affected transcripts and mapped them to pathway gene sets curated in the WikiPathway 2016 (www.wikipathways.org/) database. This resulted in enrichment of transcripts in various pathways, with TOR signalling (DDIT4, MLST8, DDIT4L, MTOR) identified as the top enriched pathway (figure 1b). To further increase the robustness of our enrichment analysis, we performed an additional gene set over-representation pathway analysis using ConsensusPathDB, a meta-database that integrates different types of functional interactions from multiple data resources [11]. From this analysis, autophagy was found to be the top pathway enriched amongst the transcripts affected by the selected variants (figure $1 \mathrm{~b}$ ). This was followed by phenyl ethylamine degradation, and target of rapamycin (TOR) signalling, the top enriched pathway when mapped to WikiPathway database alone.

Despite the differences in the study design approach, our results partially overlap with the observations from six German families with sarcoidosis with no internal familial controls and with our previous study $[12,13]$. These include rare variants/genes that are implicated in calcium metabolism (ANXA5, CDH23, $D M X L 2, T R P V 2$ ), and those involved in cell growth, survival and migration (such as PTPRD, FAT atypical cadherins and KIF), highlighting the putative role of such processes in sarcoidosis.

In this study, pooling of in silico selected susceptibility variants derived from genomic subtraction of proband cases from matched internal controls in five French families (with at least two first degree 
relatives) additionally led to the identification of genes enriched in the regulation of mTOR signalling pathway and autophagy. Previously, Linke et al. [14] described a mouse model where the deletion of the upstream mTORC1-inhibitor TSC2 in macrophages led to spontaneous development of granulomas; and also showed that sarcoid granulomas from progressive-disease patients have active mTORC1 signalling. Recently, mTORC1 signalling involvement was also identified in RNA-seq gene set enrichment data from a cutaneous sarcoidosis patient [15]. Moreover, successful treatment of a sarcoidosis patient with the mTOR inhibitor rapamycin was reported previously [16]. Here, we implicate a role of mTOR signalling in familial cases of sarcoidosis. As mTORC1 is a negative regulator of autophagy, it is particularly interesting that patients in predisposed families share mutations in genes involved in autophagy and intracellular vesicular transport, such as $A T G 9 B$ and $S E C 31 B$, suggesting that cumulative defects in autophagy regulation may play a key role in sarcoidosis pathogenesis. In a previous study, we showed that some of the genes associated with mTOR regulation were also direct regulators of Racl's functional hub, an important Rho GTPase in actin remodelling and cellular mobility and inhibiting the mTOR complex [17].

One limitation of this study is that healthy relatives in families might develop sarcoidosis later in life, even if onset of the disease in familial cases is usually early. Secondly, we are aware that we have only investigated five families in this study. Future studies should perform large-scale deep sequencing, and correlate the genotypes to disease phenotypes [18] using a similar approach as we propose here, to identify molecular targets useful for the therapeutic management of sarcoidosis patients. While we acknowledge the descriptive nature of this study, this work represents the first time, to our knowledge, that a whole exome sequencing study included internal familial controls in the study cohort, focused only on a subtype of patients (non-Löfgren syndrome sarcoidosis), pooled low frequency and rare variants identified as deleterious from multiple software packages, and derived gene pathways de novo enriched from the cohort's identified variants, without comparison to known sarcoidosis candidate genes. These are study design strategies, which we put forward for future next generation sequencing studies investigating familial sarcoidosis to consider.

Alain Calender ${ }^{1,2,13}$, Clarice X. Lim ${ }^{3,13}$, Thomas Weichhart $^{3}$, Adrien Buisson ${ }^{1}$, Valérie Besnard ${ }^{4}$, Pierre Antoine Rollat-Farnier ${ }^{5}$, Claire Bardel ${ }^{5,6}$, Pascal Roy ${ }^{5,6}$, Vincent Cottin $\oplus^{7}$, Gilles Devouassoux ${ }^{8}$, Amélie Finat ${ }^{1}$, Stéphane Pinson ${ }^{1}$, Serge Lebecque ${ }^{9}$, Hilario Nunes $^{10}$, Dominique Israel-Biet ${ }^{11}$, Abderazzaq Bentaher ${ }^{2}$, Dominique Valeyre ${ }^{12}$ and Yves Pacheco ${ }^{2}$ in the frame of GSF (Group Sarcoidosis France)

${ }^{1}$ Dept of Molecular and Medical Genetics, Hospices Civils de Lyon, University Hospital, Bron, France. ${ }^{2}$ Inflammation and Immunity of the Respiratory Epithelium, Lyon 1 Claude Bernard University, EA-7426 (PI3), Pierre-Bénite, France. ${ }^{3}$ Center for Pathobiochemistry and Genetics, Institute of Medical Genetics, Medical University of Vienna, Vienna, Austria. ${ }^{4}$ Lung and Hypoxia, EA-2363, University Sorbonne Paris Cité, Paris, France. ${ }^{5}$ Dept of Biostatistics and Bioinformatics, Hospices Civils de Lyon, University Hospital, Lyon, France. ${ }^{6}$ University Lyon 1, CNRS, Laboratory of Biometry and Evolutionary Biology, UMR 5558, Villeurbanne, France. ${ }^{7}$ Dept of Pulmonology, Hospices Civils de Lyon, Louis Pradel Hospital, Lyon, France. ${ }^{8}$ Dept of Pulmonology, Hospices Civils de Lyon, Croix Rousse Hospital, Lyon, France. ${ }^{9}$ Cancer Research Center, INSERM U-1052, CNRS-5286, Lyon, France. ${ }^{10}$ Dept of Pulmonology, Avicenne Hospital, EA-2363, University Paris 13, Bobigny, France. ${ }^{11}$ Dept of Pulmonology, European Hospital Georges Pompidou, Paris, France. ${ }^{12}$ INSERM U-1272, University Paris 13 and AP-HP, Avicenne Hospital, Bobigny, France.

${ }^{13}$ These authors contributed equally to this work.

Correspondence: Alain Calender, Dept of Molecular and Medical Genetics, Hospices Civils de Lyon, University Hospital Claude Bernard Lyon 1, Pathology Center, Hospital Groupe East, 69677 Bron cedex, France. E-mail: alain.calender@ chu-lyon.fr

Received: Nov 052018 | Accepted after revision: April 142019

Conflict of interest: A. Calender has nothing to disclose. C.X. Lim has nothing to disclose. T. Weichhart has nothing to disclose. A. Buisson has nothing to disclose. V. Besnard has nothing to disclose. P.A. Rollat-Farnier has nothing to disclose. C. Bardel has nothing to disclose. P. Roy has nothing to disclose. V. Cottin reports personal fees for lecturing and consultancy, and non-financial support for travel from Actelion, grants, personal fees for development of educational material, lecturing and consultancy, and non-financial support for travel from Boehringer Ingelheim, personal fees for consultancy from Bayer/MSD, GSK and Galapagos, personal fees for adjudication board work from Gilead, personal fees for lecturing and consultancy from Novartis, grants, personal fees for lecturing and consultancy, and non-financial support for travel from Roche, grants from Sanofi, personal fees for data monitoring committee work from Promedior and Celgene, outside the submitted work. G. Devouassoux has nothing to disclose. A. Finat has nothing to disclose. S. Pinson has nothing to disclose. S. Lebecque has nothing to disclose. H. Nunes reports grants and personal fees for consultancy from Roche/Genentech and Boehringer Ingelheim, and has acted as an investigator in a clinical trial for Sanofi and Gilead, outside the submitted work. D. Israel-Biet has nothing to disclose. A. Bentaher has nothing to disclose. D. Valeyre reports personal fees for advisory board work from Boehringer Ingelheim and Roche, personal fees for lecturing from AstraZeneca, financial support for transportation and accommodation related to scientific meetings from Boehringer Ingelheim and Roche, outside the submitted work. Y. Pacheco has nothing to disclose.

Support statement: This work was support by the Austrian Science Fund (P27701-B20 and P30857-B28), Fondation Maladies Rares (2016) and INNOVARC-DGOS (12-027-0309). Funding information for this article has been deposited with the Crossref Funder Registry. 


\section{References}

1 Pereira CAC, Dornfeld MC, Baughman R, et al. Clinical phenotypes in sarcoidosis. Curr Opin Pulm Med 2014; 20: 496-502.

2 Sakthivel P, Bruder D. Mechanism of granuloma formation in sarcoidosis. Curr Opin Hematol 2017; 24: 59-65.

3 Celada LJ, Hawkins C, Drake WP. The etiologic role of infectious antigens in sarcoidosis pathogenesis. Clin Chest Med 2015; 36: 561-568.

4 Esteves T, Aparicio G, Garcia-Patos V. Is there any association between sarcoidosis and infectious agents? A systematic review and meta-analysis. BMC Pulm Med 2016; 16: 165

5 Moller DR, Rybicki BA, Hamzeh NY, et al. Genetic, immunologic, and environmental basis of sarcoidosis. Ann Am Thorac Soc 2017; 14: S429-S436.

6 Rossides M, Grunewald J, Eklund A, et al. Familial aggregation and heritability of sarcoidosis: a Swedish nested case-control study. Eur Respir J 2018; 52: 1800385.

7 Rivera NV, Ronninger M, Shchetynsky K, et al. High-density genetic mapping identifies new susceptibility variants in sarcoidosis phenotypes and shows genomic-driven phenotypic differences. Am J Respir Crit Care Med 2016; 193: 1008-1022.

8 Pacheco Y, Calender A, Israël-Biet D, et al. Familial versus sporadic sarcoidosis: BTNL2 polymorphisms, clinical presentations, and outcomes in a French cohort. Orphanet J Rare Dis 2016; 11: 165.

9 Manolio TA, Collins FS, Cox NJ, et al. Finding the missing heritability of complex diseases. Nature 2009; 461: 747-753.

10 Judson MA, Costabel U, Drent M, et al. The WASOG Sarcoidosis Organ Assessment Instrument: an update of a previous clinical tool. Sarcoidosis Vasc Diffuse Lung Dis 2014; 31: 19-27.

11 Herwig R, Hardt C, Lienhard M, et al. Analyzing and interpreting genome data at the network level with ConsensusPathDB. Nat Protoc 2016; 11: 1889-1907.

12 Kishore A, Petersen B-S, Nutsua M, et al. Whole-exome sequencing identifies rare genetic variations in German families with pulmonary sarcoidosis. Hum Genet 2018; 137: 705-716.

13 Calender A, Rollat Farnier PA, Buisson A, et al. Whole exome sequencing in three families segregating a pediatric case of sarcoidosis. BMC Med Genomics 2018; 11: 23.

14 Linke M, Pham HTT, Katholnig K, et al. Chronic signaling via the metabolic checkpoint kinase mTORC1 induces macrophage granuloma formation and marks sarcoidosis progression. Nat Immunol 2017; 18: 293-302.

15 Damsky W, Thakral D, Emeagwali N, et al. Tofacitinib treatment and molecular analysis of cutaneous sarcoidosis. N Engl J Med 2018; 379: 2540-2546.

16 Manzia TM, Bellini MI, Corona L, et al. Successful treatment of systemic de novo sarcoidosis with cyclosporine discontinuation and provision of rapamune after liver transplantation. Transpl Int 2011; 24: e69-e70.

17 Besnard V, Calender A, Bouvry D, et al. G908R NOD2 variant in a family with sarcoidosis. Respir Res 2018; 19: 44.

18 Schupp JC, Freitag-Wolf S, Bargagli E, et al. Phenotypes of organ involvement in sarcoidosis. Eur Respir J 2018; 51: 1700991 